\title{
An Improvement of Performance Handover in Worldwide Interoperability for Microwave Access using Software Defined Network
}

\author{
Fatima Laassiri ${ }^{1, \text { a }}$, Mohamed Moughit ${ }^{2, \mathrm{~b}}$, Noureddine Idboufker ${ }^{3, \mathrm{c}}$ \\ a, bIR2M Laboratory, FST, Univ Hassan UH1- Settat, Morocco \\ ${ }^{b}$ EEA\&TI Laboratory, FST, Univ Hassan, Mohammedia, Morocco \\ ${ }^{b}$ National Schools of Applied Sciences Khouribga, Univ Hassan 1, UH1- Settat, Morocco \\ ${ }^{c}$ National School of Applied Sciences, Univ Cadi Ayyad Marrakech, Morocco
}

\begin{abstract}
The WiMAX network designates in common language of a set of standards and techniques of the world of Wireless Metropolitan Area Networks (WMAN). The standard IEEE 802.16 or WiMAX allows the wireless connection of companies or individuals over long distances at high speed. WiMAX provides an appropriate response for some rural or hard-to-reach areas, which today lack access to Broadband Internet for cost reasons. This technology aims to introduce a complementary solution to the Digital Subscriber Line (DSL) and cable networks on the one hand, and to interconnect WiFi hotspots, on the other hand WiMAX is mainly based on a star topology although mesh topology is possible. Communication can be done in Line of Sight (LOS) or not (NLOS).Software Defined Network (SDN) is a new network paradigm used to simplify network management. It reduces the complexity of network technology.The following article aims to expose a simulation implemented under Omnet4.6++, to improve Handover performance and QoS (End-to-end delay, latency, jitter, MoS and lost packet), by implemented an algorithm in SDN controller. The simulation is tested in WIMAX architecture, and results have been collected from two scenarios with and without SDN controller to proof that this algorithm is more preferment to guarantee a better QOS in Handover.
\end{abstract} $4.6++$

Keywords-WiMAX; SDN; QoS; handover; openflow; OMNeT

\section{INTRODUCTION}

WiMAX (Worldwide Interoperability for Microwave Access) [1] was standardized in 2002 under the name 802.16. Its goal was to cover high-speed white areas [2]

WiMAX technology is synonymous with better transfer rates, lower latency, better availability and efficiency, but not compatible with existing $2 \mathrm{G}$ and $3 \mathrm{G}$ systems, a major pitfall. In addition, switching from a $3 \mathrm{G}$ network to a $4 \mathrm{G}$ network is often more difficult, and sometimes problematic.

It is distinguished by the vast extent of its Internet coverage, because it is a very broad band. Besides, there are several types of high-speed Internet coverage (eg 3G, 4G, LTE, satellite and WiMAX). It offers another aspect of how conventional remote systems have been described, due to the effects of innovation on the public and its impact on the environment.
The mechanical points of interest of WiMAX are central points of unprecedented WiMAX radio change. It works in the field of security and quality. It has an open access base focused on IP access. This innovation can be used for various applications. It is a scalable remote correspondence system capable of providing high-speed remote access with high data rate of Fourth-Generation (4G) over a long separation in a point-to-multipoint and visible or an unobservable path condition.

This article presents an improvement the $\mathrm{HO}[3]$ and the QoS, " End-to-end delay, latency, jitter, the number of lost packets, and the MOS ", by the implementation a new algorithm that it allows the Software Defined Network (SDN)[4] controller to better improve the performance of all WiMAX network architectures using OMNeT4.6 ++.

\section{StATE OF THE ART}

WiMAX Wireless Microwave Metro Networks that are useful in broadband access, as a central innovation in the IEEE 802.16 reference group, advancing in $4 \mathrm{G}$. With the current presentation of portability management systems in the IEEE 802.16e standard, it is currently competing with current and future ages of remote advances to provide ubiquitous recording arrangements. Nevertheless, the establishment of a decent versatile structure depends to a large extent on the ability to make quick and consistent transfers regardless of the situation of the building being sent. Since the IEEE has characterized the mobile WiMAX MAC layer transfer (IEEE 802.16e) administration structure, the WiMAX Forum Network Working Group (NWG) [5] is working on improving upper layers, or the path to commercialization of an undeniable WiMAX versatility structure, which it is tasked to investigate the difficulties. This focuses on potential research issues related to the transfer into the current and future WiMAX portability structure. An examination of these issues in the MAC, Network and Cross Layer situations are presented alongside the exchange of distinctive answers to these difficulties.

According to research done by the "Mojtaba Seyedzadegan" on the overview of WiMAX, its architecture deals with the supply of data. It is entirely based on IEEE 
802.16. It supports two things. One is the alternative broadband cable and the other is DSL.

Subscriber Station (SS)[6] gives the link to WiMAX. Many websites are used to provide the tool used for this method. But this is not a complete view of the tools available as certified that they are set in mobile internet devices and various private labelled tools, laptops.

It assembles Wi-Fi[7] activities because of the same wireless network. It also gives network connectivity to businesses and at home without the help of external devices. For this, it uses WiMAX carriers. It is scaled a few kilometers. It varies at the scale of a city. It is given with a subscriber unit. It helps the customer to connect to the Internet and other accesses. Physical Layer It's worth mentioning that both LTE and WiMAX use Orthogonal Frequency Division Multiple Access (OFDMA)[8] in the downlink, but they differ in the uplink. WiMAX continues to use OFDMA, while LTE's[9] approach is more advanced. Using OFDMA is power inefficient, but it's tolerable in the downlink because the power amplifier is placed at the base station (or at the e-Node-B in 3GPP terminology). At the base station, power is available, and the many mobile terminals share the extra complexity. However, in the uplink, the transmissions start from mobile devices, which are battery powered. The mobile devices are also constrained because they must be low cost to enable mass deployment. 3GPP[10] specifications thus propose a reduced Peak to Average Power Ratio (PAPR) transmission scheme for the uplink signal. This scheme is called Single Carrier Frequency Division Multiple Access (SCFDMA). This makes it easier for the mobile terminal to maintain a highly efficient signal transmission using its power amplifier. The LTE uplink signal achieves this property and saves power without degrading system flexibility or performance. [11]

\section{STANDARD OF 802.16 WIMAX}

The next table (Table 1) presents the standard of 802.16 WiMAX [12]

TABLE I. CURRENT AND FEATURE STANDARD OF 802.16 WiMAX

\begin{tabular}{|l|l|}
\hline Standard & Description \\
\hline $802.16 .2-2004$ & Recommended practice \\
\hline $802.16-2009$ & Air interface for fixed and mobile \\
\hline $802.16 \mathrm{~h}-2010$ & Iproved Coexistence mechanism \\
\hline p802.16n & Higher Reliability Networks (In progress) \\
\hline p802.16k-2007 & Bridging of 802.16 \\
\hline $802.16 \mathrm{j}-2009$ & Multi hop relay \\
\hline $802.16 \mathrm{~m}-2011$ & Advanced air interface with high data rate \\
\hline p802.16p & Support machine to machine Apllication( In progress) \\
\hline
\end{tabular}

\section{PROBLEM AND SOLUTION}

In recent years, the SDN [13] plays an important role because of its flexibility and ease of transport. WiMAX is the promising $4 \mathrm{G}$ network to meet the needs of customers. It is a telecommunication technology, which provides softwaredefined data for several distances from a point-to-point link to all cell-type accesses, and it allows the connection between mobile and fixed networks. The coverage area of WiMAX is highly compared to other technologies, among different technologies; it offers good support and good stability.

WiMax refers to certain types of Wireless Local Area Networks (WLANs) [14], which use 802.16 specifications. It is easily used by companies.

Due to the growing demand, users have ubiquitous access to wireless services, which has led to the deployment of forced use of this wireless access technology such as WiMax. It offers a level of quality, within range, but the problem is that the number of devices is increasing which reduces Handover's travel time performance and QoS in terms of end-to-end delay, latency, jitter, number of lost packets, and MOS, for all these reasons, This work proposes as a solution to implement the SDN technology for WiMax to better optimize their performance through the creation of a new algorithm under SDN controller.

\section{ADVANTAGES OF WiMAX}

WiMAX is typically used as an alternative to dedicated links and Internet access of all kinds for the following applications

- It is the best solution for suburban and urban areas, where there is a wired technology problem, for large cities, this technology can also be implemented to meet all high-speed requirements;

- possibility of reusing a frequency dedicated to a BTS to increase the capacity of the system, also to support hundreds of users;

- frequency allocation is done on a sectoral basis when the number of users increases;

- networks with high transmission speeds for voice and data;

- connect to the Internet peripheral neighborhoods or suburban cities;

- inter-site private networks for companies;

- security and surveillance that may include video over IP applications;

- regional wireless networks with data and voice applications for industry and transport;

- wireless communications integrating VoIP;

- temporary deployments: Religious events, construction sites, relief infrastructure on a natural disaster;

- that it is a wireless technology with a signal range of a few hundred meters and a maximum bit rate of about $11 \mathrm{Mbits} / \mathrm{s}$, WiMAX has a technology that manages bandwidth. Thus, a user that he performs an operation, that it requires a lot of resources (High-quality video conference for example) will have a large bandwidth;

- low WiMAX allows faster deployment without the need for heavy civil engineering work; 
- it allows high-speed wireless internet connectivity over long distances;

- it can serve multiple clients at a time;

- one signal despite the obstacles;

- private inter-site networks for companies;

- perspective of nomadism;

\section{DisAdVANTAGES OF WiMAX}

- Debit is shared between users of the same central antenna;

- obligation to serve WiMAX base stations through a collection network (optical fiber, radio link, etc.);

- requirement to have a licence: Only licensees are able to deploy WiMAX networks;

- need to have a high point: To ensure the best possible coverage, the transmitter must be placed on a high point (pylon, water tower, etc.);

- it must first obtain a licence from a public authority;

- to have optimal distances and speeds, transmitters and receivers must be in "line of sight";

- it will be able to cross only small obstacles like a tree or a house but, the signal is unable to pass through hills or large buildings;

- methods for the implementation of $4 \mathrm{G}$ with and without SDN

\section{METHODS AND IMPLEMENTATION OF WIMAX WITH AND WITHOUT SDN USING OMNET $4.6++$}

Software Defined Metropolitan An Network (SDMAN)[13], it is a standard network that is designed to provide broadband access for a large area. There is more flexibility and service. It can be used in both the licensed frequency bands (10-66GHZ) and the unlicensed band (11GHZ). Therefore, it is the best technology for the system designed as long as SDN whereas the good performance and the effective cost. So, that he faces different types of problems in mobile communication. A frequency higher than $10 \mathrm{GHz}$ is required and if visibility is reduced, a frequency below $10 \mathrm{GHz}$ is essential.

It allows a broadband access service, which helps the customer to take advantage of low-cost Internet options. In general, it is a software-defined technology that operates on a frequency between 2 and $66 \mathrm{GHz}$. In addition, it provides data rates up to $75 \mathrm{Mb} / \mathrm{s}$. Thus, it becomes the backbone of the many software defined communication services. In order to increase the applications of WIMAX.

This work expresses an improvement of the QoS "end-toend delay, latency, jitter, the number of lost packets, and the MOS " through the intecration of a new algorithm that it allows to chang SDN controller policy for handle handover in the WiMAX network, by two scenarios, the first (Figure 1) expresses WiMAX without SDN and the second (Figure 2) is based on the implemented of SDN program, using OMNeT4.6++.

Scenario 2 (Figure 1): WiMAX without SDN: Is implemented with 10 base stations and with a frequency of 2.4 GHZ.

Scenario 1 (Figure 2): WiMAX with SDN: Is implemented with 10 base stations with a frequency of $2.4 \mathrm{MHZ}$, an SDN controller, and an OpenFlow switch.

You will find attached the parameters of WiMAX used: Frequency: $2400 \mathrm{MHz}$.

\author{
BSAntennaHeigh \\ BasicEnergyLowBatteryThreshold: 0.1 \\ Mobile Rx height: 1.5 meter \\ Effective radius: $4.34 \mathrm{Km}$ \\ Forest/Trees, Offset-loss: $10 \mathrm{~dB}$ \\ Open, Offset-loss: $17 \mathrm{~dB}$ \\ Buildings, Offset-loss: $-4 \mathrm{~dB}$ \\ DL Carrier bandwidth: 9x3.3MHzusingFR 1/3 \\ DL Carrier bandwidth: 3 x $10 \mathrm{MHz}$ using FFR 1/1 \\ Bearers for DL and UL: 1 \\ CINR requirement: $4.9 \mathrm{~dB}$ \\ Services for DL and UL: 1 \\ UL Carrier bandwidth: $1 \times 3.3 \mathrm{MHz}$ \\ Suburban, Offset-loss: $-3 \mathrm{~dB}$ \\ Noise Figure : $7 \mathrm{~dB}$ \\ Horizontal Beam width of CPE: 360 \\ Noise Figure : $4 \mathrm{~dB}$ \\ Receiver Sensitivity: $-109.7300 \mathrm{~dB}$ \\ Control Activity: $20 \%$ \\ BS antenna: Kathrein80010 \\ BS antenna gain: $18 \mathrm{~dB}$ \\ Horizontal beam width of BS antenna: $60^{\circ}$ \\ Vertical beam width of BS antenna: $6^{\circ}$ \\ Feeder loss: $2 \mathrm{~dB}$ \\ Prediction resolution: $10 \mathrm{~m}$ \\ Intra-Site fading correlation coefficient: 0.8 \\ Inter-Site fading correlation coefficient: 0.5
}

WiMAX lies in it's simplicity of implementation. It will take only two antennas to connect two remote networks, where it would have been miles of optical fiber wired.

The WiMAX client must have a receiver (a built-in chip or a CPE: Customer Premise Equipment) and be within the scope of a transmitter. The transmission between the customer and his hot spot WiMAX is said in "No Line Of Sight" (NLOS), that it is to say that the customer is not in direct view with the antenna. Indeed, buildings or vegetation found in cities "force" the signal to be diverted through the use of OFDM frequency modulation. 


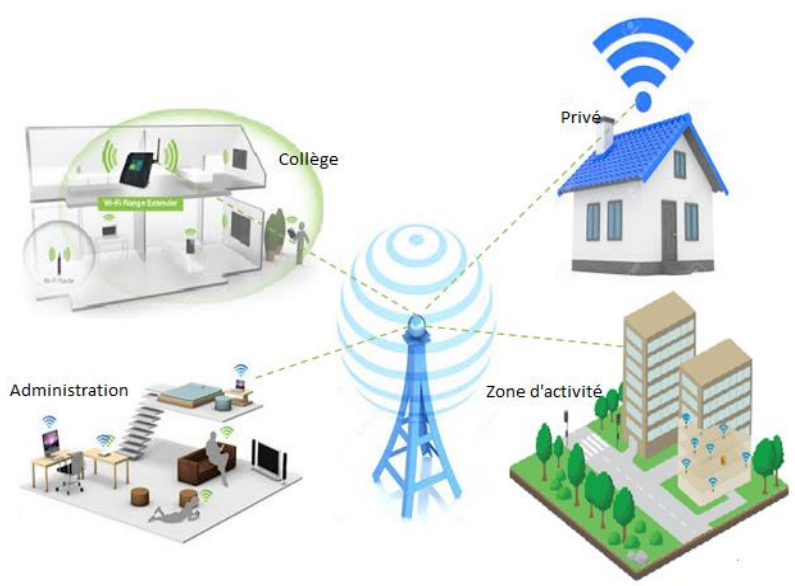

Fig. 1. WiMAX without SDN.

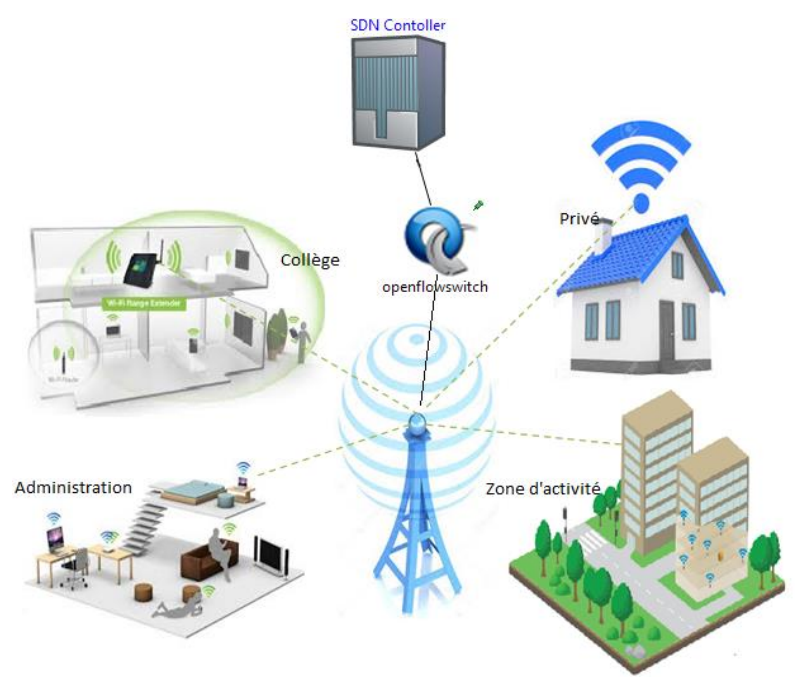

Fig. 2. WiMAX with SDN.

In a network, the collection consists of connecting the access points (WiFi or DSLAM hot spots) thus ensuring the connection with the Internet. This mechanism is called the backhauling of hotspots. Unlike the service, the collection is done in "Line Of Sight" (LOS), thanks to WiMAX transmitters placed high enough.

VIII. RESULTS AND DISCUSSION OF SIMULATION IN QOS CRITERIA UNDER WIMAX WITH AND WITHOUT SDN USING OMNET $4.6++$

This section presents the QoS performance results for WiMAX using SDN, such as end to end delay, latency, jitter, lost packet, and MOS.

\section{A. End to end Delay under WiMAX with and without SDN}

Figure 3 shows that the end to end delay in the WiMAX scenario without SDN with a higher value (26 ms) compared to the SDN based scenario that, it has a reliable delay $(0.6 \mathrm{~ms})$, that it expresses the implemented of SDN to WiMAX, it has a positive impact.

\section{B. Jitter under WiMAX with and without SDN}

The jitter under figure 4, where the WiMAX scenario, that it is based on SDN is about $10 \mathrm{~ms}$, that it is lower than
WiMAX without SDN, that it has the value of $11 \mathrm{~ms}$, which results that the connection of an SDN network for WiMAX is successful.

\section{Latency under WiMAX with and without SDN}

The results of figure 5 shows that the WiMAX network latency with SDN is less (14 ms) than that of the WiMAX approach without SDN with a value of $16 \mathrm{~ms}$, which explains that the value added by the latter is beneficial

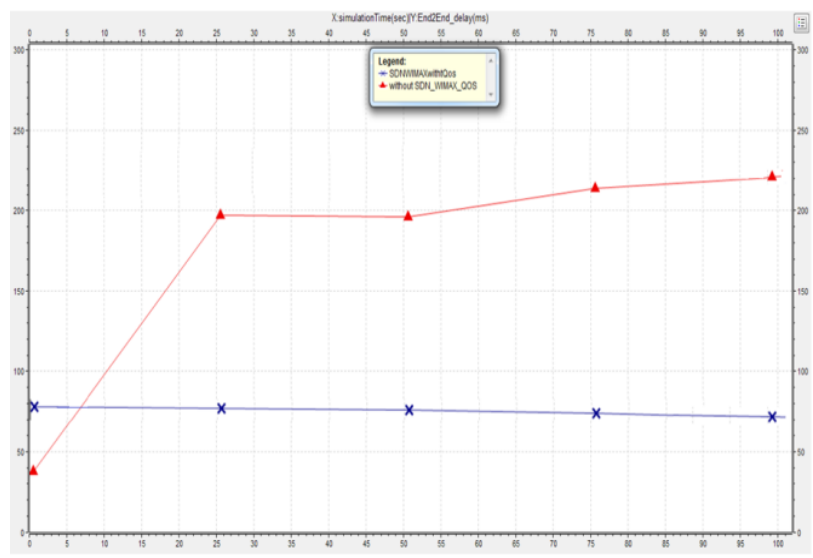

Fig. 3. End to end Delay under WiMAX with and without SDN.

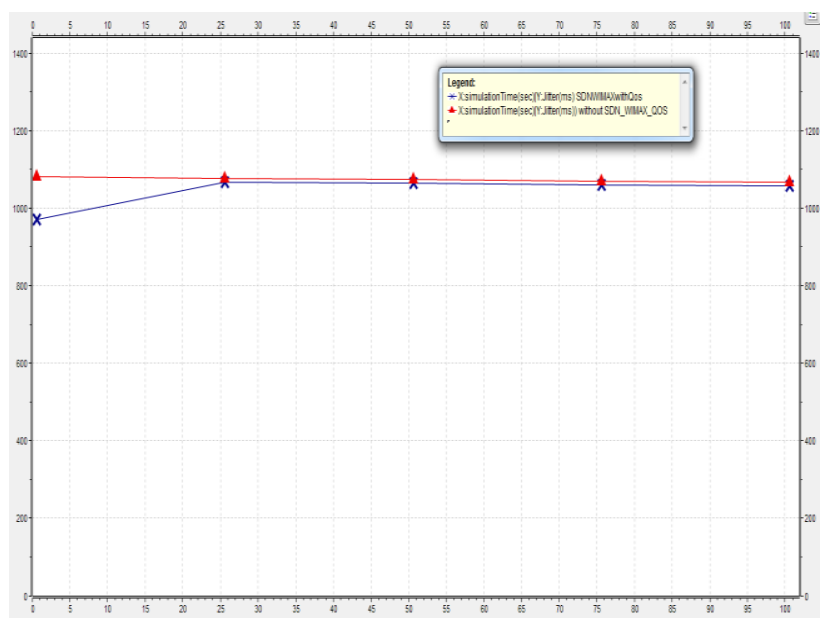

Fig. 4. Jitter under WiMAX with and without SDN.

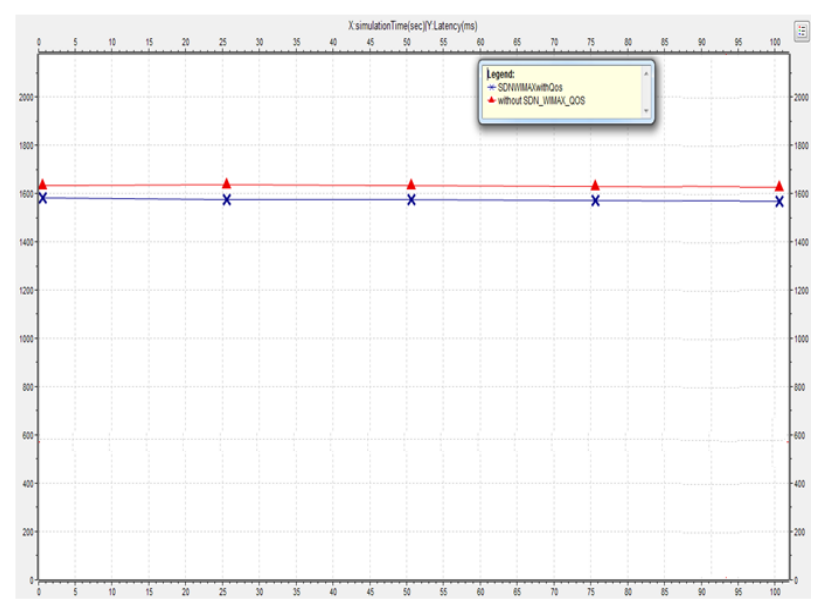

Fig. 5. Latency under WiMAX with and without SDN. 


\section{Packets Lost under WiMAX with and without SDN}

The number of packets lost in the WiMAX approach without SDN is 7\%, which is higher compared to the WiMAX approach with SDN that it is about 5\%. This shows that the impact of adding SDN for the WiMAX network is totally favorable, as shown in figure 6.

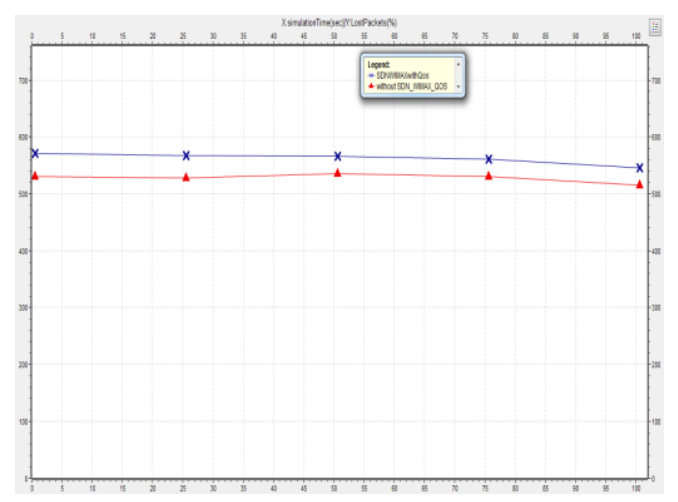

Fig. 6. Packets lost under WiMAX with and without SDN.

\section{E. MOS under WiMAX with and without SDN}

Figure 7 shows that the MOS offered by the WiMAX approach without SDN is 1 , whereas the WiMAX based approach with SDN is about 2.3, which presents an indicator of the increases in the quality of the WiMAX voice transmission.

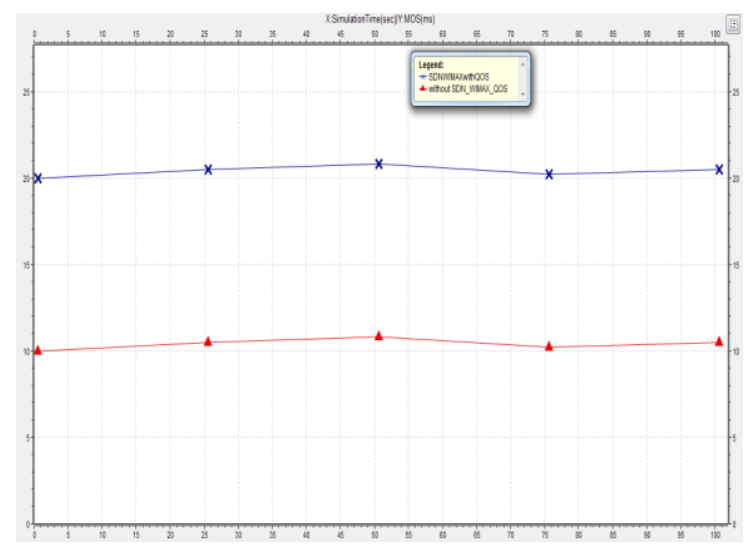

Fig. 7. MOS under WiMAX with and without SDN.

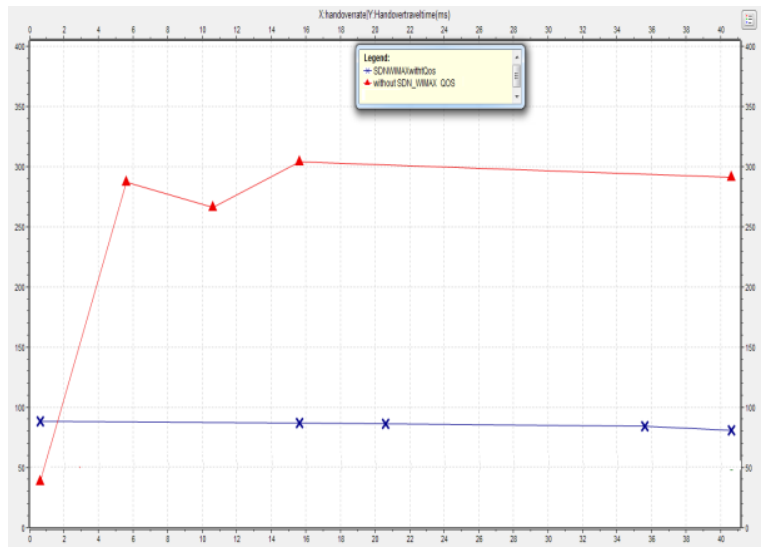

Fig. 8. Handover under WiMAX with and without SDN.

\section{RESUlts AND Discussion OF Simulation IN HANDOVER CRITERIA UNDER WIMAX WITH AND WITHOUT SDN}

Figure 8 shows through two scenarios that the transfer time of the nodes in a WiMAX network with SDN is stable during all communication with a low time $(0.7 \mathrm{~ms})$, whereas without SDN, it has the value of $30 \mathrm{~ms}$, then SDN makes it possible to minimize the time of movement from one node to another by the centralization at the controller level.

\section{CONCLUSION}

SDN controller is the best way that allows developers to change Network control with programming efficient algorithms. In this article we proof that our algorithm in comparison with existing SDN controller is very suitable for WIMAX network to more guarantee QOS in Handover.

\section{REFERENCES}

[1] Hung-Yu Wei, S. GangulyR, Iz mailov, "Interference-aware IEEE 802.16 WiMax mesh networks', IEEE 61st Vehicular Technology Conference.

[2] http://4glte.over-blog.com/article-le-wimax-ou-le-lte-3-5-ghz86329750.html, Jun 2018.

[3] Allan Borges Pontes, Diego dos Passos Silva, Jose Jailton, "Handover management in integrated WLAN and mobile WiMAX networks", IEEE Wireless Communications : Volume: 15, Issue: 5, October 2008.

[4] Fatima LAASSIRI, Mohamed MOUGHIT, Noureddine IDBOUFKER, "Evaluation of the QoS Parameters in Different SDN Architecture using Omnet 4.6++', IEEE, March 2018.

[5] Ronny Yongho Kim, Jin Sam Kwak, Kamran Etemad, "WiMAX femtocell: requirements, challenges, and solutions', IEEE Communications Magazine, Volume: 47, Issue: 9, September 2009.

[6] James A. Hutchison, IV Rotem Cooper, Paul T. Williamson, "'Subscriber station with dynamic multi-mode service acquisition capability", App/Pub Number : US10254143, 2005.

[7] Brian Ferris, Dieter Fox, Neil Lawrencet, "WiFi-SLAM Using Gaussian Process Latent Variable Models', IJCAI-07 2480, 2007.

[8] Ala'a Al-Habashna; Octavia A. Dobre; Ramachandran Venkatesan ; Dimitrie C. Popescu , "Second-Order Cyclostationarity of Mobile WiMAX and LTE OFDM Signals and Application to Spectrum Awareness in Cognitive Radio Systems', IEEE Journal of Selected Topics in Signal Processing, Volume: 6, Issue: 1, Feb. 2012.

[9] David Martín-Sacristán, Jose F. Monserrat, Jorge Cabrejas-Peñuelas, Daniel Calabuig, Salvador Garrigas, Narcís Cardona, "On the Way towards Fourth-Generation Mobile: 3GPP LTE and LTE-Advanced”, Springer 03 August 2009.

[10] Douglas N. Knisely, Takahito Yoshizawa Frank Favichia Standardization of femtocells in 3GPP', I EEE Communications Magazine (Volume: 47 , Issue: 9 , September 2009.

[11] Zakhia Abichar and J. Morris Chang, Chau-Yun Hsu, "WiMAX vs. LTE: Who Will Lead the Broadband Mobile Internet?', 1520-9202/10/\$26.00 C 2010 IEEE, IT Pro May/June 2010.

[12] Mohammed Torad, Ahmed El Qassas, Hadia Al Henawi, “Comparison between LTE and WiMAX based on system level simulation using OPNET modeler (release 16)', IEEE, June 2011.

[13] Steven Gringeri; Nabil Bitar; Tiejun J. Xia, “Extending software defined network principles to include optical transport', IEEE Communications Magazine, Volume: 51, Issue: 3, March 2013.

[14] A. Kumar; E. Altman; D. Miorandi; M. Goyal, "New insights from a fixed point analysis of single cell IEEE 802.11 WLANs', Proceedings IEEE 24th Annual Joint Conference of the IEEE Computer and Communications Societies, 22 August 2005. 